\title{
Fast and Non-Catalytic Growth of Transparent \& Conductive Graphene-Like Carbon Films on Glass at Low Temperature
}

\author{
Roberto Muñoz ${ }^{1}$ and Cristina Gómez-Aleixandre ${ }^{1}$. \\ ${ }^{1}$ Surfaces \& Coatings Dept, Materials Science Institute of Madrid, ICMM-CSIC, Madrid, 28049, Spain

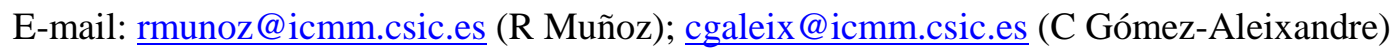

\begin{abstract}
This article presents the synthesis and systematic study on graphene-like carbon thin films directly grown on commercial glass by using remote-ECR Plasma Assisted CVD. The fabrication process is extremely rapid and performed on to 2 inch scale dielectric substrate at relatively low temperature $\left(<550^{\circ} \mathrm{C}\right)$ without using metal catalyst. This method avoids damaging and expensive transfer processes of graphene based films and improves compatibility with current fabrication technologies. Nanoestructural characterization by TEM indicates the formation of layered graphene-like carbon material. Raman Spectroscopy shows that the film consists of nanocrystals with a mean domain size close to $2 \mathrm{~nm}$, probably interconnected by amorphous material. These graphene-like carbon based films are transparent and conductive. Functional optoelectric characterization of these films confirms their high transparency over $95 \%$ and relative high conductivity around $5 \mathrm{k} \Omega$, exceeding the properties of non-doped small domain graphene based films grown at low temperatures reported by far.
\end{abstract}




\section{Introduction}

Graphene, the one atom thick layer of carbon, uniquely combines supreme physical properties that justify its nickname of a "miracle material". A number of these properties -mechanical stiffness, strength, elasticity, electrical and thermal conductivity- are extreme. [1,2] Moreover, the combination of these properties suggests that graphene could replace current materials in existing applications rapidly. This versatility could justify the common inconveniences of switching to a new technology in industrial processes. One example of this is the combination of transparency and conductivity that will find use, for example, in photovoltaic solar cells or flexible electronics as a cheap transparent electrode. [3]

Although, some of these superior properties have been achieved only for the highest quality mechanically exfoliated samples, [4] other methods of synthesis are rapidly being improved. Large area high quality, polycrystalline and single crystalline graphene are now being grown by chemical vapor deposition (CVD) on copper with promising results for many applications, despite the fact that transfer processes to functional substrates are required. [4,5] At present, this process is expensive owing to large energy consumption at the typical synthesis temperatures around $1000^{\circ} \mathrm{C}$ and because underlying metal has to be removed. In this scenario, the game changing breakthrough would be the development of processes to rapidly deposit high quality graphene layers on arbitrary substrates, at low temperature. [6]

In recent times, many approaches have been developed attempting to solve these aspects. Parallel routes have been tested by many groups in this area, that include low temperature synthesis on metals by plasma assisted CVD, direct synthesis on desired dielectric substrates, commonly at high temperature, without plasma technology and finally the combination of both, namely, direct synthesis on desired substrates at low temperature by means of plasma assisted deposition. Up to now, the main CVD technologies that have been used in graphene synthesis include, among others, radio frequency plasma enhanced CVD (RF-PECVD) [12,13], microwave plasma CVD (MPCVD) [7-11] and Electron Cyclotron Resonance CVD (ECRCVD)[29].

First, for low temperature synthesis on metals by plasma assisted techniques, high quality graphene has been grown by MPCVD on $\mathrm{Cu}$ foils at $700^{\circ} \mathrm{C}$ in less than two minutes [7] and between $450^{\circ} \mathrm{C}-750^{\circ} \mathrm{C}$ on $\mathrm{Ni}$ [8] 
being the quality of these films comparable to those grown by thermal CVD. Large area roll-to-roll approach to grow graphene on $\mathrm{Cu}$ foils ( $294 \mathrm{~mm}$ wide) and $\mathrm{Al}$ foils at $300^{\circ} \mathrm{C}-400^{\circ} \mathrm{C}$ by surface wave plasma assisted CVD has been also performed using $\mathrm{CH}_{4} / \mathrm{H}_{2} / \mathrm{Ar}$ mixtures. [9,10] Graphene films with few layer structure have been also deposited on $\mathrm{Cu}$ at temperatures as low as $240^{\circ} \mathrm{C}$ using a gas mixture of $\mathrm{C}_{2} \mathrm{H}_{2}$ and $\mathrm{Ar}$ by MPCVD.[11] These films show smaller graphene domain sizes comparing with thermal CVD graphene. Multilayer graphene has been also deposited by RF-PECVD at $500^{\circ} \mathrm{C}$ on $\mathrm{Cu}$ with acceptable qualities [12] and on $\mathrm{Ni}$ at $650^{\circ} \mathrm{C}$ with a short deposition time using traces of $\mathrm{CH}_{4} \cdot[13]$ In all these examples post transfer process is needed. In this area of research two more approaches could be included. In one hand, the synthesis at low temperature by using metal alloys acting as a catalyst.[14] On the other hand, the use of Ni [15,16] or $\mathrm{Cu}$ [17] sacrificial films at high synthesis temperatures over $1000^{\circ} \mathrm{C}$ with simultaneous evaporation of catalyst that effectively avoids post-transfer processes. Although the evaporation of the catalyst is not complete in the case of $\mathrm{Ni}$, the monolayer graphene exhibits optoelectronic properties that exceed the properties of transferred films.

A second route involves direct synthesis on desired substrates normally at elevated temperatures and without plasma assistance. Atmospheric Pressure CVD (APCVD) synthesis of monolayer or few layer graphene has been performed on $\mathrm{Si}_{3} \mathrm{~N}_{4}$ at $1000^{\circ} \mathrm{C}$ [18] and $1150^{\circ} \mathrm{C}$ [19] resulting in continuous films with dozens of $\mathrm{nm}$ sized domains and sheet resistance of $3 \mathrm{k} \Omega$ or less. Also APCVD synthesis has been used on sapphire $\left(\mathrm{Al}_{2} \mathrm{O}_{3}\right)$ between $1450^{\circ} \mathrm{C}$ and $1650^{\circ} \mathrm{C}$ with $\mathrm{CH}_{4}$ as precursor [20] -with mobilities over $2000 \mathrm{~cm}^{2} / \mathrm{V} \cdot \mathrm{s}$ for monolayer films- and at $950^{\circ} \mathrm{C}$ but with detrimental effects on electronic properties.[21] Molecular beam epitaxy (MBE) from highly ordered pyrolytic graphite (HOPG) sublimation at $1100^{\circ} \mathrm{C}$ has also been tested on sapphire but the films presented small domain size, not usual at this temperature.[22] There have also been published results of graphene grown by APCVD on $\mathrm{SiO}_{2}$ substrates at $1000^{\circ} \mathrm{C}$ with $\mathrm{CH}_{4}$ as precursor with sheet resistance around $5 \mathrm{k} \Omega$ [23], and at $1100^{\circ} \mathrm{C}$ that show outstanding results with sheet resistances around $800 \Omega$ and transmittance of $91 \%$ but in processes that take from 6 to 10 hours.[24] Few layer graphene on h-BN flakes has been also deposited at $1000^{\circ} \mathrm{C}$ by APCVD.[25] Synthesis of layered graphene flakes on $\mathrm{MgO}$ nanocrystal powder has been also carried out by low pressure CVD at temperatures between $325^{\circ} \mathrm{C}$ to $875^{\circ} \mathrm{C}$ using acetylene and cyclohexane respectively, but optoelectronic properties have not been tested and Raman spectra of these samples exhibit the overlap of broadened G and D modes.[26] In all this 
experiments the high temperatures limit the compatibility of these processes with microelectronic industrial processes.

Combining both ideas implicit above, many groups have faced the challenge of direct synthesis of graphene films on desired substrates at low temperatures by means of plasma assisted deposition. Few layer vertical graphene flakes have been synthesized by $\mathrm{MPCVD}$ at $700^{\circ} \mathrm{C}$ on various dielectric substrates (quartz, $\mathrm{SiO}_{2}$ ). [27] Although Raman spectra have shown the high quality of the films, functional characterization has not been included. Remote PECVD has been also successfully applied in the direct synthesis of few layer graphenic carbon at $550^{\circ} \mathrm{C}$ by using $\mathrm{CH}_{4}$ plasma on different dielectric substrates. [28] In this case the processes have taken a few hours and the best result on glass was a sheet resistance of $7 \mathrm{k} \Omega$ with transmittance of $85 \%$ and $40 \mathrm{k} \Omega$ on quartz with transmittance of $92 \%$. Recently a much more rapid approach by using ECR-CVD has been published.[29] The authors ensure the synthesis of graphene-like carbon films on pure $\mathrm{SiO}_{2}$, glass and quartz at low temperature $\left(400^{\circ} \mathrm{C}\right.$ to $\left.750^{\circ} \mathrm{C}\right)$ and in few minutes by using $\mathrm{C}_{2} \mathrm{H}_{4} / \mathrm{Ar}$ plasma. Although the exact synthesis temperature is not defined in each case, the published results of these films on quartz exhibit outstanding transmittance from $94 \%$ to $82 \%$ and sheet resistances around $20 \mathrm{k} \Omega$ to 7 $\mathrm{k} \Omega$. It is also proved that the sheet resistivity of the films can be decreased, by doping, down to $3 \mathrm{k} \Omega$ although the transmittance measurements are not included.

Here, we report the direct and rapid synthesis of continuous graphene-like carbon thin films on cheap commercial glass substrates at temperatures around $500^{\circ} \mathrm{C}$ by means of a modified remote ECR-CVD system using $\mathrm{CH}_{4} / \mathrm{Ar}$ gas mixtures, for the first time. The process is completed in few minutes. By using remote plasma activation, the detrimental effects of the plasma ion etching on the quality of the films are avoided. The effective activation of $\mathrm{CH}_{4}$ in the plasma is followed by optical emission spectroscopy (OES). The nanostructure, continuity and crystallinity of the films have been characterized by scanning electron microscopy (SEM), Raman spectroscopy and transmission electron microscopy (TEM). Transmittance and sheet resistance measurements show that the quality of the films exceeds noticeably the current results reported at these temperatures in this type of materials without subsequent doping. The process is reproducible and compatible with current optoelectronic fabrication technologies. These results represent a step forward to practical applications of these graphene-like carbon thin films as transparent and conductive electrodes. 


\section{Experimental}

\subsection{Film deposition}

Graphene-like carbon films have been grown in a remote ECR-CVD system using Ar to activate and stabilize the ECR plasma and $\mathrm{CH} 4$ as precursor. The system mainly consists of an ASTEX AX 2000 microwave power source, two zone chamber and a two stage pumping system. Figure 1 shows a scheme of the system, indicating the transport mechanism and kinetic stages of the growth process. After the insertion of $\mathrm{Ar}$ at the top of the chamber the first stage (1) is the transport and diffusion of Ar by forced convention to the upper zone that is rounded by the ECR magnet. The second stage (2) is the activation of the Ar neutrals by means of microwave power. The next stage is the insertion of methane through a floating distribution ring of $10 \mathrm{~cm}$ of diameter with a hole size of $1 \mathrm{~mm}^{2}$ (3) and Ar species collision with methane producing activation and dissociation mainly downstream distribution ring. After the activation process the generated species $\left(\mathrm{C}_{\mathrm{x}} \mathrm{H}_{\mathrm{y}}, \mathrm{C}_{2}, \mathrm{CH}, \mathrm{CH}^{+}, \mathrm{H}, \mathrm{H}^{+}\right.$, etc) adsorb on the substrate surface (4) where the thermal-mediated surface processes take place (5), including, surface migration, nucleation and finally film growth. The final steps are the desorption of byproducts from the surface (6), transport of byproducts by diffusion through the boundary layer and back to the main gas stream (7) and transport of byproducts by forced convection away from the deposition region (8). In this type of processes, efficiency is always much lower than $100 \%$ so a major part of initial hydrocarbon molecules together with molecular hydrogen and neutralized hydrocarbon radicals will desorb from the surface as byproducts, due to their low sticking coefficients at the conditions which the reactions take place.

Prior to the growth process the glass substrates have been annealed few minutes in Ar plasma to clean the surface and remove organic contaminants at the process temperature below $550^{\circ} \mathrm{C}$, partial pressure of $6,3 \cdot 10^{-3}$ mbar and 200W. The growth process has been then started introducing a $\mathrm{CH}_{4}$ flow lower than $1 \mathrm{sccm}$ at the same power through the distribution ring in the lower zone of the chamber. The film growth has been performed during, 2, 3, 4 and 6 minutes. After growth, the plasma has been turned off and two different post annealing treatments at $10^{-2}$ mbar pressure without plasma activation, one in $\mathrm{Ar}$ and the other in $\mathrm{H}_{2}$ atmosphere, have been tested.

Optical emission spectroscopy (OES) studies of the plasma have been performed using a EGG \& Princeton Applied research Model 1235 Digital Triple-Grating spectrograph. The system is equipped with a Diode 
Array Detector Model 1453A and a Detector Interface Model 1471A. The spectrograph is controlled by a OMA-VISION data acquisition and analysis software. The spectra have been captured 5 min after plasma ignition with an acquisition time of $10 \mathrm{~s}$. It is worthy to mention that OES spectra only accounts for approximately $5 \%$ of the actual composition of the plasma. However, it is a valuable technique to evidence the plasma activation and dissociation of the carbon precursor.

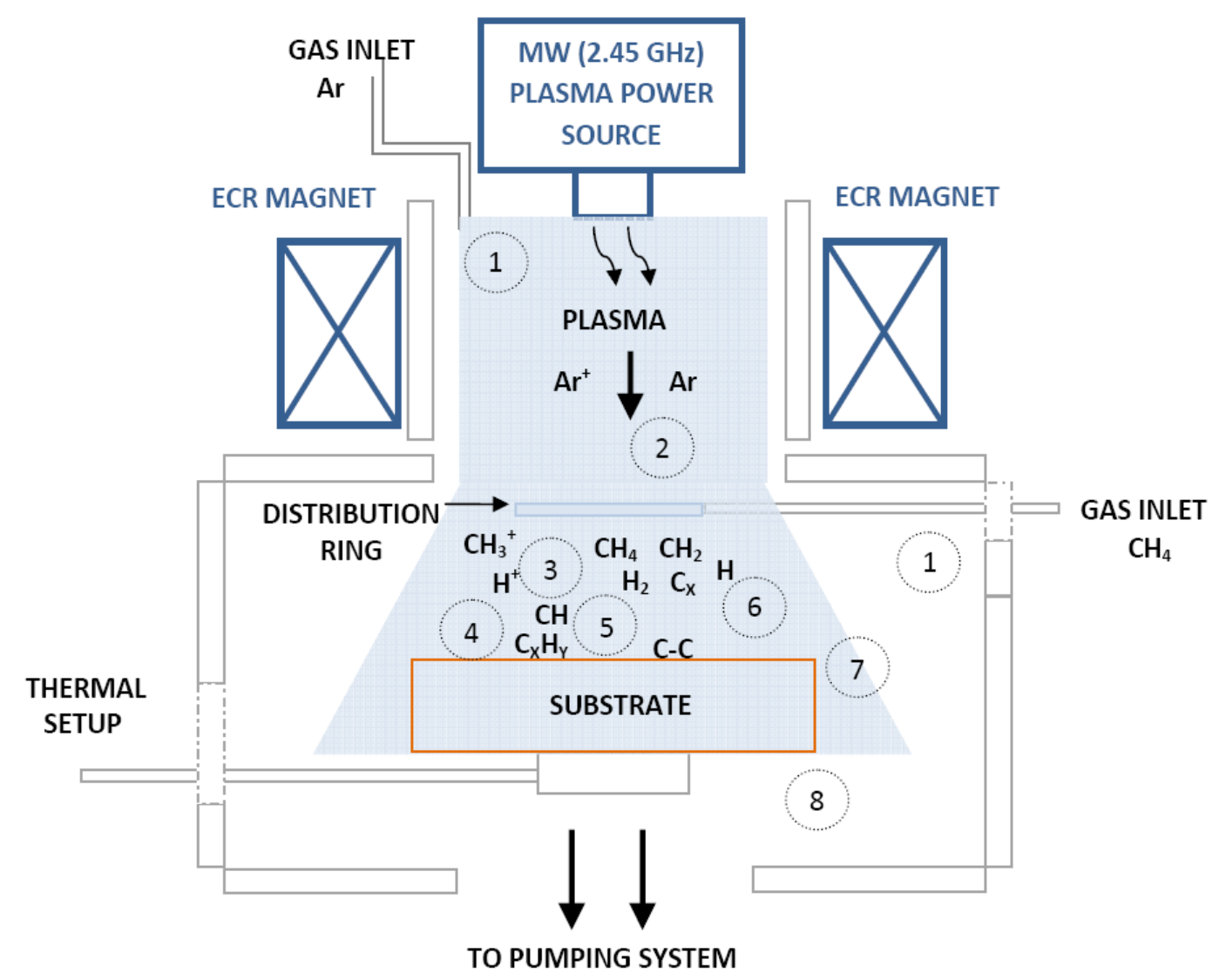

Figure 1. Schematic diagram of the ECR-CVD system. The transport mechanims and kinetics of the growth process are numbered in hollow circles as follows: 1 . Transport of reactants by forced convection. 2. Plasma activation zone. 3. Ar activated species collision with methane producing activation and dissociation mainly downstream distribution ring. 4. Adsorption of reactants on the substrate surface. 5 . Thermal-mediated surface processes, including, surface migration, nucleation and finally film growth. 6 . Desorption of byproducts from the surface. 7. Transport of byproducts by diffusion through the boundary layer and back to the main gas stream. 8. Transport of byproducts by forced convection away from the deposition region.

\subsection{Film characterization}

TEM analysis of the films has been conducted using a JEOL JEM 2100 transmission electron microscope equipped with $\mathrm{a} \mathrm{LaB}_{6}$ thermoionic electron gun. Acceleration voltage of $200 \mathrm{kV}$ has been used throughout the measurements. The TEM images have been recorded by a ORIUS CCD SC1000 (Model 832). A simple 
method has been used to prepare the samples for observation, that has not modified chemically the nanostructure of the films. The films have been scratched and the resulting small flakes and debris were deposited onto cellulose covered copper grids for observation. Before spreading out the flakes, a thin film of sputtered amorphous carbon has been also applied to the grid as a reference for electron diffraction analysis.

A Micro Raman Spectrometer (Enwave Optronics Inc., EZ-RAMAN-N series) equipped with a 40x objective has been used to provide a spot size of approximately $5 \mu \mathrm{m}$. Laser excitation is $\lambda=532 \mathrm{~nm}$, and the power level has been set at minimum to avoid heating or damage of the sample. To compare the Raman spectra of the films grown for different time periods, the integration time has been set at $10 \mathrm{~s}$ over all points on all samples. For transmittance measurements, we have used a SHIMADZU SolidSpec-3700 UV/Vis/NIR Spectrophotometer. Sheet resistance was measured using the four-probe method in a JANDEL Model RM2 universal probe. Prior to the sheet resistance measurements, the four-probe tips have been cleaned using ethanol and air dried during few minutes.

\section{Results and discussion}

\subsection{Plasma characterization}

Figure 2 shows a typical OES spectrum of the ECR-CVD plasma captured during the deposition process, where the characteristic peaks have been numbered. In the spectrum it can be observed two signals (peaks 1 and 3) at $390 \mathrm{~nm}$ and $431 \mathrm{~nm}$, assigned to $\mathrm{CH}$ species, coming from methane dissociation, which correspond to the transitions $\mathrm{B}^{2} \Sigma \rightarrow \mathrm{X}^{2}$ II and $\mathrm{A}^{2} \Delta \rightarrow \mathrm{X}^{2}$ II respectively. In addition, the peaks in the range 510-520 nm (peaks 6) have been attributed to the $\mathrm{C}_{2}$ species that usually are formed in methane discharges from the monocarbon species originated in the dissociation of methane molecules.

Also the presence of atomic hydrogen becomes evident from the detection of the signals 4,5 and $9\left(\mathrm{H}_{\gamma}, \mathrm{H}_{\beta}\right.$ and $\mathrm{H}_{\alpha}$ at 435, 487 and $657 \mathrm{~nm}$ ). Similarly, the peaks detected in the range 580-630 nm (peaks 8) have been associated with hydrogen molecules in the plasma, necessarily coming also from methane molecules dissociation. Finally, the signals located at $420 \mathrm{~nm}$ (peak 2) and $603 \mathrm{~nm}$ (peak 7) as well as the most intense ones in the zone of highest wavelength (peaks 10) have been assigned to argon species. 


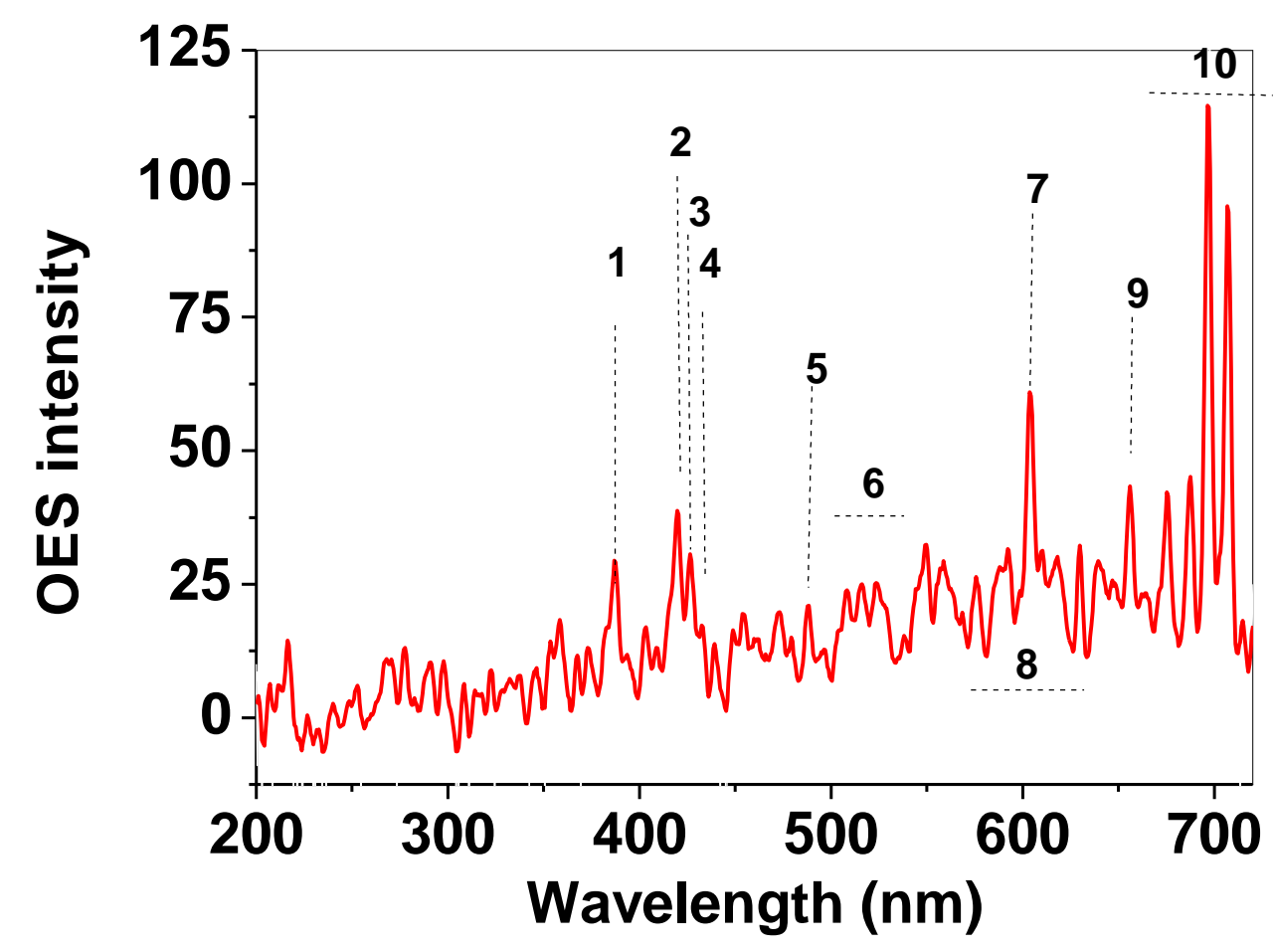

Figure 2. Optical Emission Spectroscopy spectrum of the remote ECR plasma acquired during the growth process.

Following previous studies about ECR discharges in argon/methane/hydrogen gas mixtures,[30] we consider that Ar atoms, directly introduced in the excitation zone, are activated by electron impact. Downstream the excitation zone, methane molecules go into the chamber by means of a ring located between this excitation zone and the substrate holder. Therefore, the activated argon species collide with methane molecules subsequently producing their dissociation and/or activation. The detection of $\mathrm{CH}, \mathrm{C}_{2}$ species, and atomic and molecular hydrogen suppose an effective dissociation of the $\mathrm{C}$ source molecules by the ECR discharge. It is noteworthy the complexity of the OES spectra and the weak intensity of the signals corresponding to carbon containing species, due to the low concentration required for the growth of graphene layers. Finally, the characteristic emission peaks of atomic $\mathrm{H}$ are fairly low, even more than $\mathrm{CH}$ characteristic peak at 390 $\mathrm{nm}$, that indicate low atomic $\mathrm{H}$ content in the plasma. This could have important implications in the nanostructure of the deposited films in relation with their amorphous carbon content. 


\subsection{Nanostructural characterization}

Figure 3 shows the optical photographs of the graphene-like carbon based films grown on glass substrates at different times. In the figure the enhancement in the light absorption with the deposition time is easily observed. Figure 4 shows scanning electron microscopy (SEM) images of the graphene-like carbon based films grown for 6 minutes comparing the two different postannealing processes. The shown films are continuous. A little less roughness is seen in Figure 4a due to the more regular mechanical etching of Ar atoms during annealing. The $\mathrm{H}_{2}$ treatment (see Figure $4 \mathrm{~b}$ ) seems to have stronger chemical character due to the preferential $\mathrm{H}_{2}$ interaction with residual amorphous material. The bright spots have been associated with small graphene nanodomains.

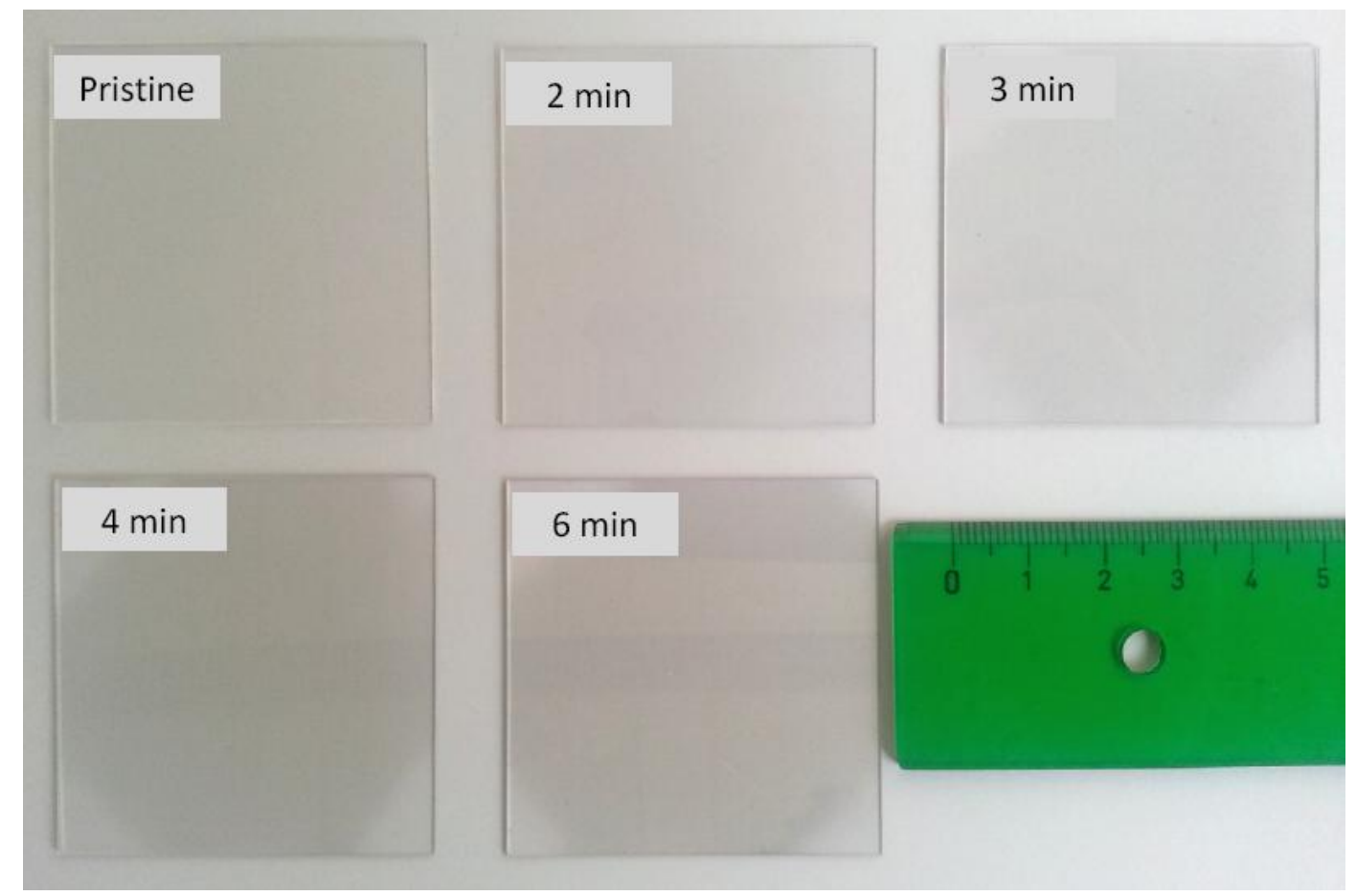

Figure 3. Optical photograph or graphene-like carbon based films with different thicknesses obtained for different growing periods. 
Para ver esta película, debe

disponer de QuickTime ${ }^{T M}$ y de

un descompresor.

Figure 4. SEM images of the graphene-like carbon based films grown at 6 minutes with a) Ar annealing and b) $\mathrm{H}_{2}$ annealing.

The crystalline structure and layered character of the films have been observed by transmission electron microscopy (TEM) and simultaneous electron diffraction measurements in two samples grown for 2 and 6 minutes with $\mathrm{H}_{2}$ post annealing. Sputtered amorphous carbon on the TEM grids has been also used as an electron diffraction model to compare its amorphous structure to the structure of our films from the electron diffraction analysis. Figure 5a, 5b and 5c show TEM images of the samples. Layered structures at the free standing edges are seen in figure $5 \mathrm{a}$ for the thinnest sample $(2 \mathrm{~min})$, since graphene tends to roll up at free edges in a similar way as carbon nanotubes during sample preparation and transfer to TEM grids. High resolution image in figure $5 \mathrm{~b}$ also shows the layered character of the film. At the bottom of the image up to 4 monolayers can be seen at the rolled up edge corresponding to $1-2 \mathrm{~nm}$ thickness. Figure $5 \mathrm{c}$ shows a similar image of the film deposited during 6 minutes. In this case up to ten stacked layers can be seen confirming the higher thickness of the sample up to 4-5 nm. Simultaneously, electron diffraction patterns have been captured during the observation of both samples. Previously a diffraction pattern of amorphous carbon has been obtained as a reference to compare to those of the samples that is shown in Figure 5d. Figures $5 \mathrm{e}$ and $5 \mathrm{f}$ show the electron diffraction patterns of the two samples grown for 2 and 6 minutes respectively. Both figures clearly show a well defined diffraction ring, typical signature of nanocrystalline material. This diffraction rings are much more defined respect to the amorphous carbon one. Even in the figure $5 \mathrm{f}$ two bright diffused spots can be seen that indicates effectively that the film consists of stacked layers. The 
distance between these spots and the transmitted beam is around $2.6 \mathrm{~nm}^{-1}$ that may correspond to a interlayer separation of $0.38 \mathrm{~nm}$. This fact indicates the turbostratic character of the films.

Para ver esta película, debe disponer de QuickTime ${ }^{\mathrm{TM}}$ y de un descompresor.

Figure 5. TEM images and electron diffraction analysis of the films at different growth times. a) Low magnification image showing the layered character of the film grown in 2 minutes. b) and c) Zoom images of the films grown at 2 and 6 minutes respectively showing flake edges. d) Electron diffraction analysis of typical amorphous carbon. e) and f) Electron diffraction analysis around the same zone of the films grown at 2 and 6 minutes respectively. 
Further characterization of the structure of the films has been carried out by means of Raman spectroscopy.

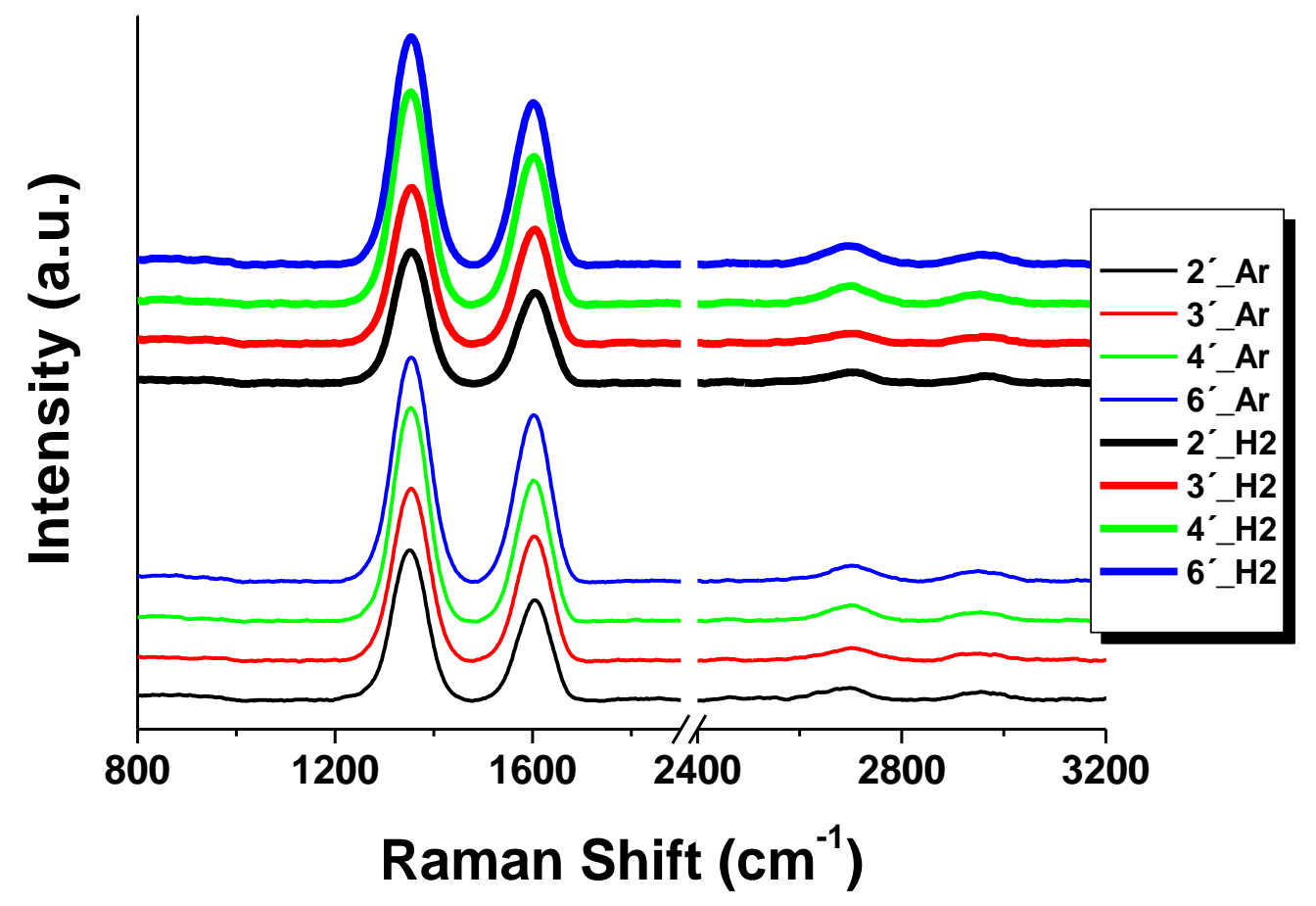

Figure 6. Raman spectra of the samples at different growth times with Ar or $\mathrm{H}_{2}$ post annealing treatment.

Figure 6 includes the Raman spectra of all the samples for different growth times and post annealing treatments. All the Raman spectra in figure 6 appear quite similar. For all the samples, the characteristic peaks of carbon $\mathrm{sp}^{2}$ bonding are observed in the Raman spectra: $\mathrm{D}$ peak centered at near $1350 \mathrm{~cm}^{-1}, \mathrm{G}$ peak near $1600 \mathrm{~cm}^{-1}$ and $2 \mathrm{D}$ peak centered at $2700 \mathrm{~cm}^{-1}$. The $\mathrm{G}$ peak shift from the standard position at $1580 \mathrm{~cm}^{-1}$, is really due to the appearance of the $\mathrm{D}^{\prime}$ peak at $1620 \mathrm{~cm}^{-1}$ that merges in the $\mathrm{G}$ peak for small grain sizes. The main difference that has been clearly observed among the spectra is the enhanced intensities of all the characteristics peaks $D, G$ and $2 D,\left(I_{D}, I_{G}\right.$ and $\left.I_{2 D}\right)$ with the deposition time. No differences regarding the post annealing treatments have been detected. The principal characteristics of the spectra are the fairly low $2 \mathrm{D}$ peak and a high $I_{D}$, common features of graphene based films with nm size domains. [29] In this case, the high $\mathrm{I}_{\mathrm{D}}$ and its large full width at half maximum (FWHM) $\left(\sim 60 \mathrm{~cm}^{-1}\right)$ could be related to both nanodomain boundaries and the residual amorphous carbon, that we consider connecting the nanodomains. Following the seminal work of Tuinstra and Koening (TK) [31] and the three-stage model of Ferrari and Robertson [32, 33, 34], the $\mathrm{I}_{\mathrm{D}} / \mathrm{I}_{\mathrm{G}}$ relation can be used to estimate the size of $\mathrm{sp}^{2}$ bonded clusters or in plane interdefect distances. 
For the studied samples, we detect a broadening in G and D peaks, the G peak well centered around 1600 $\mathrm{cm}^{-1}$ and the $\mathrm{I}_{\mathrm{D}} / \mathrm{I}_{\mathrm{G}}$ around 1.4, which corresponds to nanocrystalline samples at the limit of the first and second stages of the Ferrari and Robertson model $[32,33,34]$, when the $I_{D} / I_{G}$ is proportional to $L_{a}{ }^{2}$. Then, an average domain size around 2-3 nm may be inferred. Finally it is important to note that the $2 \mathrm{D}$ peak is also well centered at $2700 \mathrm{~cm}^{-1}$. According to the degree of stacking order in graphite systems, the 2D band should be composed of two asymmetric peaks that can be fitted by using two Lorentzians centered approximately at $2687 \mathrm{~cm}^{-1}$ and $2727 \mathrm{~cm}^{-1}$ when $3 \mathrm{D}$ order occurs.[35] The 2D peak in all the spectra of figure 6 is almost totally symmetric, typical characteristic for films with turbostratic structure. This fact confirms the turbostratic structure of the films, already proposed from TEM images and electron diffraction analysis.

\subsection{Optical and electrical characterization.}

The optical and electrical properties of the films have been also evaluated. Figure 7 shows the optical transmittance and sheet resistance of the samples grown at different process times and with different postannealing processes. As already shown by other authors, [11,29,36,37] the transmittance $(\mathrm{T})$ and sheet resistance $\left(R_{\mathrm{s}}\right)$ of the graphene deposit diminish as the deposition time increases, which also presumes a higher thickness. In addition, as can be observed, there is to some extent a different behavior of the transmittance of the films depending on the post- annealing treatment. 

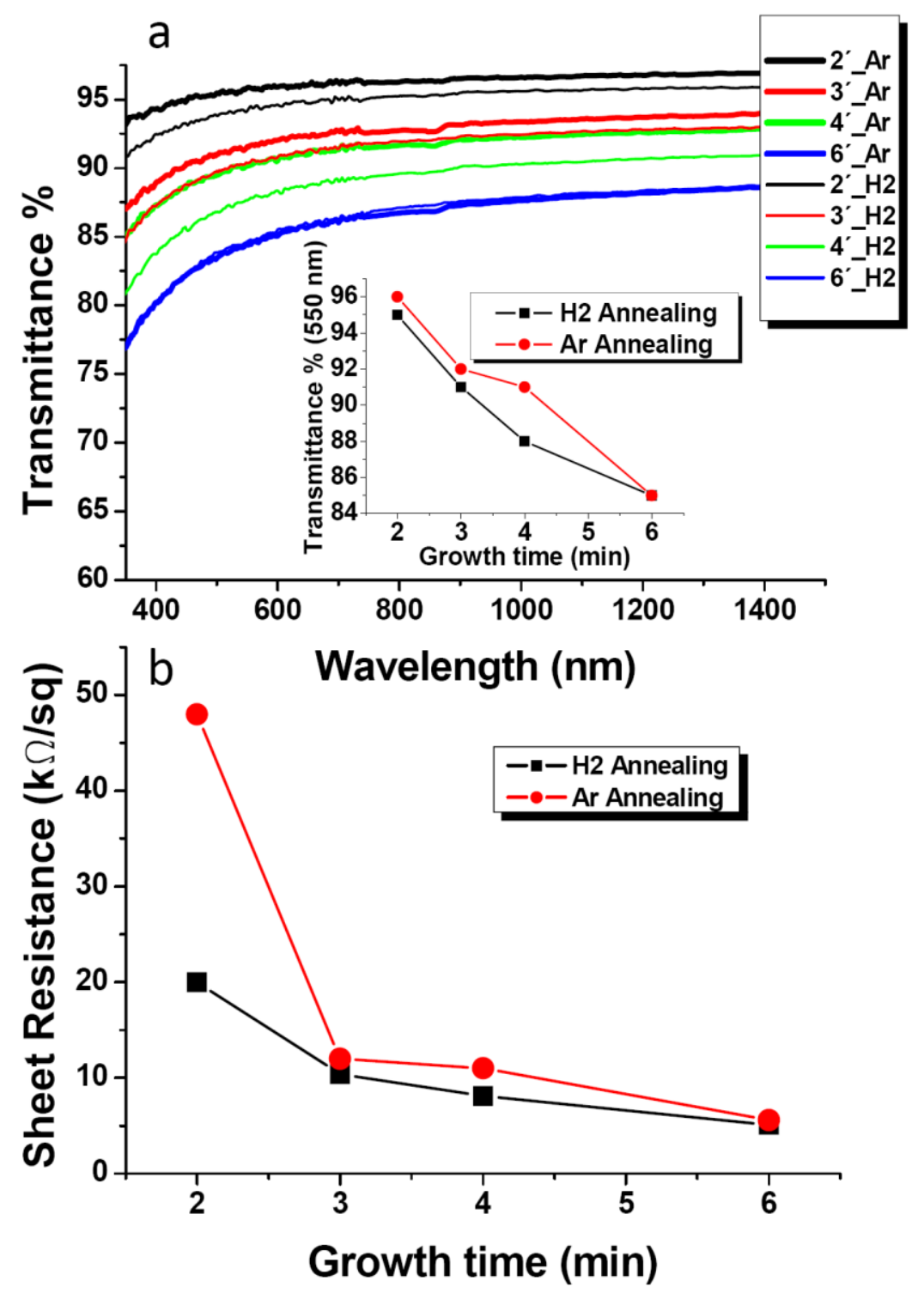

Figure 7. a) Optical transmittance of graphene-like carbon based films grown on glass at different process times and post annealing processes. The inset compares the transmittance of the films at 550 nm. b) Sheet resistance of the films.

In Figure $7 \mathrm{a}$, is observed a slightly lower transmittance of the films annealed in $\mathrm{H}_{2}$, particularly for short deposition times. In the thinnest films the different character of the etching, more mechanical in the case of Ar atoms and more chemical due to $\mathrm{H}_{2}$ that preferentially interacts with residual amorphous material, may result in different thinning and surface morphology of the films as seen by SEM. This effect may be considered negligible for the thickest films. Figure $7 \mathrm{~b}$ shows the variation of the sheet resistance of the films with the deposition time. Again the difference in the sheet resistance is more dramatic in the thinnest sample 
( 2 min process). For the other samples the annealing influence on the resistance is lower despite the fact that the samples annealed with Ar are always more resistive. The best values for the thinnest sample annealed in $\mathrm{H}_{2}$ are transmittance of $95 \%$ and sheet resistance around $19.8 \mathrm{k} \Omega \mathrm{sq}^{-1}$. In contrast, more functional samples with $85 \%$ transmittance and around $5 \mathrm{k} \Omega \mathrm{sq}^{-1}$ have been deposited in 6 min.

It is relevant to take into account one fundamental question here. The high transmittance of $95 \%$ observed for the thinnest films, would correspond to two layers of turbostratic graphene films synthesized by thermal CVD [3] but our TEM characterization shows up to four layers. This fact may be due to certain amorphous carbon content in the films. A high amorphous content is not actually expected because of the high conductivity of the films and the nanoestructural characterization. However, the amorphous phase may induce a double effect, namely, it may reduce the conductivity and simultaneously enhance somehow the transparency of the deposit, as in some DLC systems [38,39,40] Another key question is that not only is important the domain size of the nanocrystals but also how they connect together.

In transparent conducting films, both physical properties $\left(T\right.$ and $R_{s}$ ) are controlled by the optical and DC conductivities respectively and the following relationship, Equation 1, can be assumed for a thin conducting film [37]:

$$
T=\left(1+\frac{Z_{0}}{2 R_{\mathrm{s}}} \frac{\sigma_{\mathrm{Op}}}{\sigma_{\mathrm{DC}}}\right)^{-2}
$$

being $\mathrm{Z}_{0}$ the impedance of the free space $(377 \Omega)$ and $\sigma_{\mathrm{DC}}$ and $\sigma_{\mathrm{Op}}$ the electrical and optical conductivity of the material, respectively. Thus, the variation of $T$ and $R_{s}$ is controlled by the conductivity ratio $\sigma_{\mathrm{DC}} / \sigma_{\mathrm{Op}}$. Notice that high values of this ratio correspond to wanted good properties for this type of transparent conducting layers (high $\mathrm{T}$ and low $\mathrm{R}_{\mathrm{s}}$ ).

In this study, where it has been seen that $\mathrm{T}^{-1 / 2}-1$ varies nearly linearly with $\mathrm{Z}_{0} / 2 \mathrm{Rs}$ (not shown), [37] we can apply surely the above proposed relationship (Equation (1)) between T y Rs. In this way, the calculated conductivity ratio $\sigma_{\mathrm{DC}} / \sigma_{\mathrm{OP}}$, gives valuable information about the characteristics of the graphene deposits, allowing us to compare them with deposits grown by other authors. In Figure 8, the calculated $\sigma_{\mathrm{DC}} / \sigma_{\mathrm{Op}}$ for different graphene samples, including those grown in this work, is shown. As can be seen, $\sigma_{\mathrm{DC}} / \sigma_{\mathrm{Op}}$ of our 
samples reaches a high value compared with most of them. Solely, higher values of the conductivity ratio have been obtained for either the ECR CVD samples deposited by Medina et al,[29] at temperatures between $400^{\circ}$ and $750^{\circ} \mathrm{C}$ or for the doped graphene samples obtained by reduction of graphene oxide, during long time periods (more than $2 \mathrm{~h}$ ).[41] As outstanding result, it must be highlighted that in this study, high values of $\sigma_{\mathrm{DC}} / \sigma_{\mathrm{Op}}$ are achieved when non-doped graphene layers are deposited in a few minutes $(<5 \mathrm{~min})$ by a transfer-free ECR-CVD process at low temperature $\left(<550^{\circ} \mathrm{C}\right)$ without using metal catalyst. Hence, the ECRCVD appears as an adequate method for growing graphene-like layers with relatively low electrical resistivity. Moreover, it is noteworthy that subsequently, the resistivity of the layers may be dramatically reduce by means of the proper doping, giving as a result transparent and highly conducting graphene films, with straight application in Optoelectronics or solar cells technology.

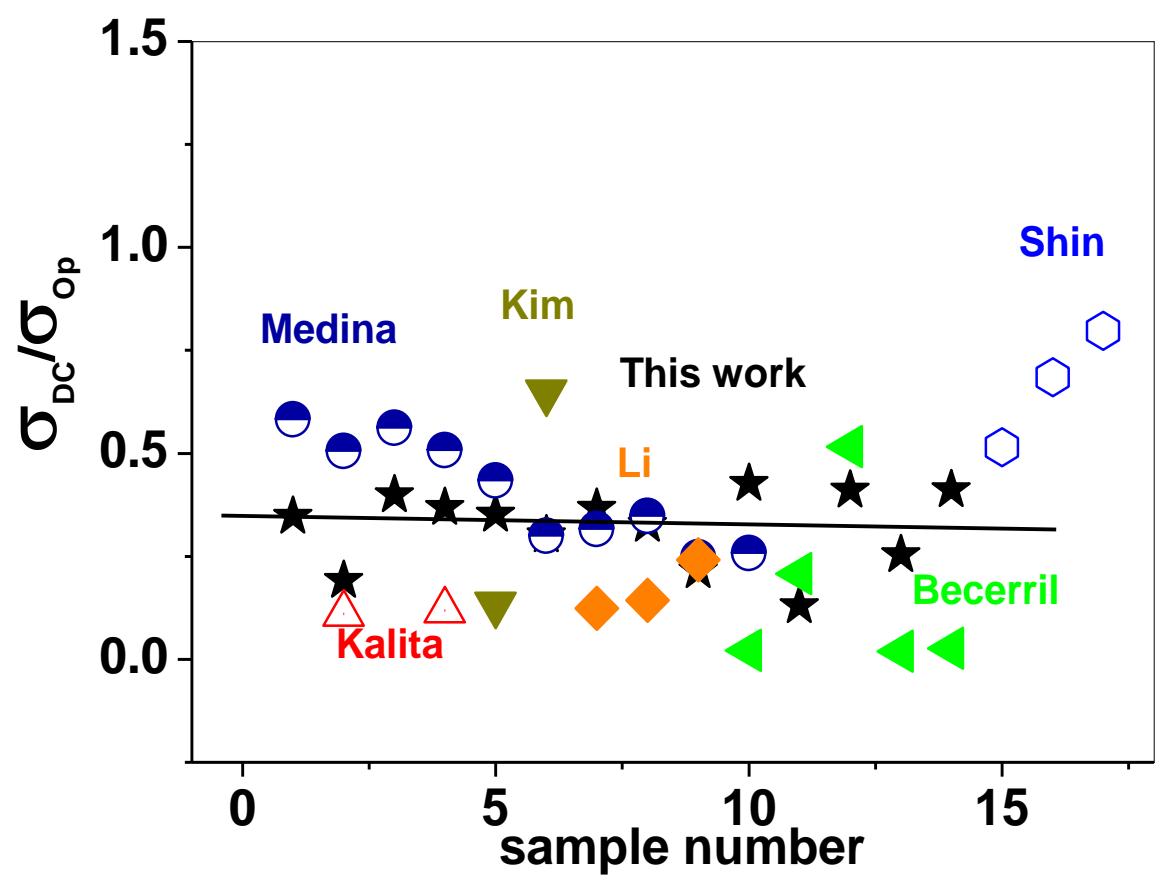

Figure 8. Calculated $\sigma_{\mathrm{DC}} / \sigma_{\mathrm{Op}}$ for graphene films deposited by different authors and methods.

\subsection{Graphene-like carbon films growth mechanism}

Transition metals, mainly $\mathrm{Cu}$ and $\mathrm{Ni}$ are widely employed as catalyst for the graphene growth by thermal CVD.[42,43] The standard CVD growth begins with catalyst-assisted hydrocarbon dissociation, followed by adsorption of $\mathrm{CHx}$ radicals on metal surface, thermal assisted diffusion, polymerization and graphitization.[44] Therefore, a key question is whether the catalytic decomposition of methane has to be 
taken into account even when using silicon oxide based substrates or not, since the hydrocarbon dissociation is likely completed by plasma activation. CVD growth of carbon nanotubes using nanoparticles as a catalyst has become possible in thermal systems.[45-48] According to some authors [29,49], in this case, the catalytic dissociation taking place on the substrate surface would be mediated by the $\mathrm{SiC}$ formation. However, it is known that $\mathrm{SiC}$ formation through solid-state carbon reduction of silica is only accomplished at high temperatures over $800^{\circ} \mathrm{C}$, clearly above our process temperature. To provide more information to this question we have studied the evolution of Raman spectrum captured from the thinnest sample ( 2 min growth) comparing it to that captured from a pristine glass substrate (not included). The broad and asymmetric characteristic peak of $\mathrm{SiC}$ near $800 \mathrm{~cm}^{-1}$ has not been detected at all and both spectra have similar appearance. No other spectroscopic analysis to confirm Raman spectra has been performed, but based on the low synthesis temperature and the fast nucleation process (obviously less than 2 minutes), we believe that there is no appreciable catalytic effect during our growth process. Instead, we propose that the surface chemistry takes place in a series of steps. Firstly, $\mathrm{C}_{\mathrm{x}} \mathrm{H}_{\mathrm{y}}, \mathrm{CH}_{\mathrm{x}}$ and $\mathrm{C}_{2}$ radicals with low $\mathrm{H}$ content, generated in the plasma and detected by OES, adsorb and subsequently diffuse over the surface. The carbon monomers and dimmers with low hydrogen content are much more likely to adsorb due to the higher sticking coefficients. Moreover, carbon dimmers are energetically more favourable to diffuse on the surface than monomers. [44] This diffusion process is followed by a second step that involves a variable degree of short chain polymerization that is mainly dependent on the temperature although the other thermodynamic parameters are also important. The stability and mobility of this short polymer chains on the surface depends on their chain length, the amount of hydrogen not released during the polymerization reaction and the surface itself. The surface morphology and roughness have a critical role in the nucleation of this species along with the temperature of the surface. Finally, the temperature used in this work is sufficient to activate the thermal release of the remaining hydrogen and the subsequent graphitization of this short chain carbon species following a van der Waals type heteroepitaxial growth.[20,44,50,51]

\section{Conclusions}

We have demonstrated the direct growth of graphene-like carbon based films on commercial glass substrates up to 2 inches wide. The process is based on remote ECR-CVD plasma assisted deposition, being this a novel configuration not used hitherto. Complete process is accomplished rapidly at low temperature resulting 
in films free of impurities and with optical and electrical properties exceeding published results by far. Nanostructural studies by SEM, TEM and Raman spectroscopy reveal the layered and nanocrystalline character of the films being the nanocrystallites probably interconnected by a small amount of amorphous material. Instead of the catalytic effect, the new growth process is performed by hydrocarbon radicals that adsorb, diffuse and nucleate on the surface in a kind of van der Waals type heteroepitaxial growth. This method may pave the way for graphene applications in transparent conductive electrodes and other optoelectronic devices.

\section{Acknowledgements}

The authors appreciate the financial support of the Consolider-Ingenio project (CSD2008-00023), funded by the Spanish Ministry of Science and Innovation (MICINN). Also, the research leading to these results has received funding from the European Union Seventh Framework Programme under grant agreement n604391 Graphene Flagship.

\section{References}

[1] Geim A K and Novoselov K S 2007 Nature. Mater. 6, 183.

[2] Castro Neto A H, Guinea F, Peres N, Novoselov K S and Geim A K 2009 Rev. Mod. Phys. 81, 109.

[3] Bae S, Kim H, Lee Y, Xu X, Park J S, Zheng Y, Balakrishnan J, Lei T, Kim H R, Song Y I, Kim Y, Kim K S, Özyilmaz B, Ahn J H, Hong B H and Iijima S 2010 Nature. Nanotech. 5, 574.

[4] Novoselov K S, 2004 Science. 306, 666-669.

[5] Li X, Cai W, An J, Kim S, Nah J, Yang D, Piner R, Velamakanni A, Jung I, Tutuc E, Banerjee S K, Colombo L and Ruoff R S 2009 Science. 324, 1312-1314.

[6] Novoselov K S, Falko V I, Colombo L, Gellert P R, Schwab M G and Kim K 2012 Nature. 490. 192.

[7] Kumar A, Voevodin A, Zemlyanov D, Zakharov D N and Fisher T S 2012 Carbon. 50, 1546-1553.

[8] Kim Y, Song W, Lee S Y, Jeon C, Jung W, Kim M, and Park C Y 2011 Appl. Phys. Lett. 98, 263106.

[9] Yamada T, Ishihara M, Kim J, Hasegawa M and Iijima S 2012 Carbon. 50, 2615-2619.

[10] Kim J, Ishihara M, Koga Y, Tsugawa K, Hasegawa M and Iijima S 2011 Appl. Phys. Lett. 98 (2), 241247. 
[11] Kalita G, Wakita K and Umeno U 2012 RSC Adv. 2, 2815-2820.

[12] Terasawa T and Saiki K 2012 Carbon. 50 (3), 869-874.

[13] Qi J L, Zhang L X and Cao J 2012 Chin. Sci. Bull. 57 (23), 3040-3044.

[14] Weatherup R S, Bayer B C, Blume R, Ducati C, Baehtz C, Schlogl R and Hofmann S 2011 Nano Lett. 11, 4154-4160.

[15] Xiong W, Zhou Y S, Jiang L J, Sarkar A, Mahjouri-Samani M, Xie Z Q, Gao Y, Ianno N J, Jiang L, Liu Y F 2013 Adv. Mater. 25, 630-634.

[16] Yan Z, Peng Z, Sun Z, Yao J, Zhu Y, Liu Z, Ajayan P M and Tour J M 2011 ACS Nano. 5 (10), 81878192.

[17] Ismach A, Druzgalski C, Penwell S, Schwartzberg A, Zheng M, Javey A, Bokor J and Zhang Y 2010 Nano Lett. 10, 1542-1548.

[18] Sun J, Lindvall N, Cole M T, Teo K B K and Yurgens A 2011 Appl. Phys. Lett. 98, 252107.

[19] Chen J, Guo Y, Wen Y, Huang L, Xue Y, Geng D, Wu B, Luo B, Yu G and Liu Y 2013 Adv. Mater. 25, 992-997.

[20] Hwang J, Kin M, Campbell D, Alsalman H A, Kwak J Y, Shivaraman S, Woll A R, Singh A K, Henning R G, Gorantla S, Rümmeli M H and Spencer M G 2013 ACS Nano. 7(1), 385-395.

[21] Song H J, Son M, Park C, Lim H, Levendorf M P, Tsen A W, Park J and Choi H C 2012 Nanoscale. 4, 3050-3054.

[22] Jerng S K, Yu D S, Kim Y S, Ryou J, Hong S, Kim C, Yoon S, Efetov D K, Kim P and Chun S H 2011 J. Phys. Chem. C. 115, 4491-4494.

[23] Sun J, Lindvall N, Cole M T, Wang T, Booth T J, Boggild P, Teo K B K, Liu J and Yurgens A 2012 J. Appl. Phys. 111, 044103.

[24] Chen J, Wen Y, Guo Y, Wu B, Huang L, Xue Y, Geng D, Wang D, Yu G and Liu Y 2011 J. Am. Chem. Soc. 133, 17548-17551.

[25] Ding X, Ding G, Xie X, Huang F and Jiang M 2011 Carbon. 49, 2522-2525. 
[26] Rümmeli M H, Bachmatiuk A, Scott A, Börrnert F, Warner J H, Hoffman V, Lin J, Cuniberti G and Büchner 2010 ACS Nano. 4(7), 4206-4210.

[27] Malesevic A, Vitchev R, Schouteden K, Volodin A, Zhang L, Van Tendeloo G, Vanhulsel A and Van Haesendonck C 2008 Nanotech. 19, 305604.

[28] Zhang L, Shi Z, Wang Y, Yang R, Shi D and Zhang G 2011 Nano Res. 4(3), 315-321.

[29] Medina H, Lin Y C, Jin C, Lu C C, Yeh C H, Huang K P, Suenaga K, Robertson J and Chiu P W 2012 Adv. Func. Mater. 22, 2123-2128.

[30] Manuel Camero, Ph. Degree Thesis, "Capas de Carbono y Nitruro de Carbono Nanoestructuradas. Estudio de los Procesos de Síntesis asistidos por plasma” Universidad Autónoma de Madrid, June, 2006.

[31] Tuinstra F and Koenig J L 1970 J. Chem. Phys. 53, 1126 doi:10.1063/1.1674108

[32] Ferrari A C and Robertson J 2000 Phys. Rev. 61, 14095.

[33] Ferrari A C and Robertson J 2001 Phys. Rev. B. 64, 075414.

[34] Ferrari A C 2007 Solid State Communications.143, 47-57.

[35] Cancado L G, Takai K, Enoki T, Endo M, Kim Y A, Mizusaki H, Speziali N L, Jorio A and Pimenta M A 2008 Carbon.46(2), 272-275.

[36] Kim K S, Zhao Y, Jang H, Lee S Y, Kim J M, Kim K S, Ahn J H, Kim P, Choi J Y, Hong B H 2009 Nature Lett. 457, 706-710.

[37] De S, Coleman J N 2010 ACS Nano. 4(5), 2713-2720.

[38] Sahoo S, Pradhan S S, Bhavanasi V and Pradhan S K 2010 Surface \& Coatings Technology. 204, 28172821.

[39] Dwivedi N, Kumar S, Ishpal, Dayal S, Govind, Rauthan C M S and Panwar O S 2011 Journal of Alloys and Compounds. 509, 1285-1293.

[40] Aryal H R, Adhikari S, Ghimire D C, Kalita G and Umeno M 2008 Diamond \& Related Materials. 17, $680-683$. 
[41] Shin H J, Kim K K, Benayad A, Yoon S M, Park H K, Jung I S, Jin M H, Jeong H K, Kim J M, Choi J Y and Lee Y H 2009 Adv. Func. Mater. 19, 1987-1992.

[42] Reina A, Thiele S, Jia X, Bhaviripudi S, Dresselhaus M S, Schaefer, J A and Kong J 2009 Nano Res. 2, 509-516.

[43] Li X et al 2010 Nano Lett. 10, 4328-4334.

[44] Muñoz R and Gómez-Aleixandre C, 2013 Chem. Vapor. Dep. DOI: 10.1002/cvde.201300051.

[45] Takagi D, Hibino H, Suzuki S, Kobayashi Y and Homma Y 2007 Nano Lett. 7, 2272.

[46] Liu B, Ren W, Gao L, Li S, Pei S, Liu C, Jiang C and Cheng H M 2009 J. Am. Chem. Soc. 131, 2082.

[47] Bachmatiuk A et al 2009, ACS Nano. 3, 4098.

[48] Yenilmez E, Zhang H, Zhang L, Deng Z, Moler K A 2011 Nanosci. Nanotechnol. Lett. 3, 669.

[49] Page A J, Chandrakumar K R S, Irle S and Morokuma K 2011, J. Am. Chem. Soc. 133, 621.

[50] Koma A, Ueno K and Saiki K 1991 J. Cryst Growth. 111, 1029-1032.

[51] Koma A 1992 Thin Solid Films. 216, 72-76. 